\title{
Cysticercosis Neck: A Rare Presentation
}

\author{
Sunil Kumar, SP Agarwal, Malti Kumari
}

\begin{abstract}
Cysticercosis is a parasitic infection caused by the larvae of the tapeworm Taenia solium. Neurocysticercosis is the most common form of presentation. Extraneural lesions are solitary, rare and small in size which usually involves subcutaneous tissue, skeletal muscles and eyes. Human cysticercosis can occur after ingestion of pork tapeworm eggs, through feco-oral route by ingesting fecally contaminated food or by autoinfection. Solitary extraneural cysticercosis lesions may mimic other soft tissue lesions; this is the reason it is important to consider cysticercosis as a differential diagnosis during evaluating of patients with soft tissue lesions. We report a case of cysticercosis presenting as a large soft cystic swelling in the supraclavicular region of the neck. The diagnosis was suspected after ultrasonography and confirmed by microscopic examination.
\end{abstract}

Keywords: Cystic neck swelling, Cysticercosis, Extraneural.

How to cite this article: Kumar S, Agarwal SP, Kumari M. Cysticercosis Neck: A Rare Presentation. Int J Head and Neck Surg 2013;4(1):55-56.

Source of support: Nil

Conflict of interest: None

\section{INTRODUCTION}

A neck swelling have several differential diagnosis depending on age of a patient, location, duration of symptom, the clinical course and characteristics. A swelling in posterior triangle of neck in a middle aged adult is a common presentation of nasopharyngeal carcinoma, lymphoma, metastatic lymph nodes, tubercular lymphadenitis and other rare chronic infective diseases. Cysticercosis in humans is caused by the larvae of the tapeworm T. solium which is dead end host. It can manifest as either neural or extraneural forms. Extraneural forms commonly involve eyes, skeletal muscles and subcutaneous tissue in form of a single or multiple and submucosal or subcutaneous firm nodules. In literature, head and neck manifestation of cysticercosis is reported as soft tissue swelling at submental area, cheek and oral tongue. ${ }^{1,2}$ We report a case of cysticercosis presenting as a large cystic mass in the supraclavicular region of the neck which is very rare in extracranial sites. The purpose of this case report is to consider cysticercosis as a differential diagnosis when examining patients with soft tissue swelling apart from being a rare entity.

\section{CASE REPORT}

A 30-year-old man presented in our outpatient department as left side neck swelling for 1 year. Patient had no history of trauma, fever, pain or any other constitutional symptoms. Examination revealed $8 \times 6 \mathrm{~cm}$ size swelling at left side posterior triangle of neck. The swelling was soft in consistency, fluctuant, nontender and brilliantly transluminant (Fig. 1). There were no other palpable swellings in any other part of the body with normal other ear, nose and throat examination. Diagnostic needle aspiration was performed and revealed clear straw colored fluid. Clinically it was diagnosed as a case of lymphangioma. High resolution ultrasonography revealed a cystic lesion in posterior triangle of neck measuring $8.6 \times 4.0 \times 1.5 \mathrm{~cm}$ (volume $28 \mathrm{ml}$ ) with well-defined margins and few septations (Fig. 2). The patient underwent complete excision of cyst. The intraoperative findings revealed an approximately $8 \mathrm{~cm}$, cystic lesion, adherent to underlying musculature in the supraclavicular region without any intrathoracic extension. Grossly the cyst wall was thick, multiloculated and filled with clear, straw-colored fluid. Microscopic examination demonstrated cystic wall lined by thick cuticle layer and underlying loose fibrous tissue shows inflammatory cells predominantly comprising of lymphocytes, suggestive of cysticercosis (Fig. 3). The patient was advised for albendazole $400 \mathrm{mg}$ for 7 days.

\section{DISCUSSION}

Human cysticercosis occurs when they ingest $T$. solium eggs either through fecal-oral route by eating fecally contaminated food or by autoinfection. Autoinfection may occur after ingestion of eggs derived from his or her own feces or if eggs pass by reflux from the intestine into the stomach. Ingestion of encysted pork may lead to intestinal infestation of the adult tapeworm and a carrier state for the T. solium eggs. ${ }^{3,4}$ Humans are the only definitive hosts for T. solium, while pigs are the usual intermediate hosts, although dogs, cats and sheep may harbor the larval forms.

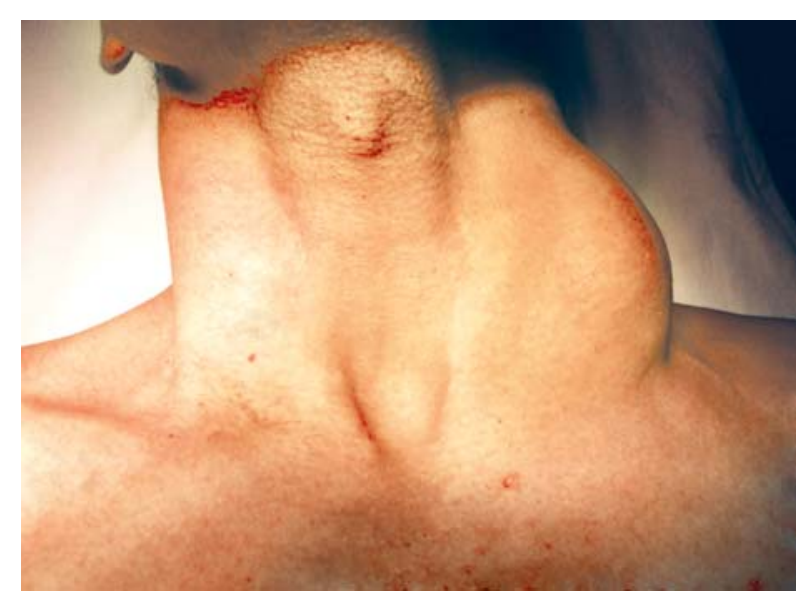

Fig. 1: Large swelling left side supraclavicular region 


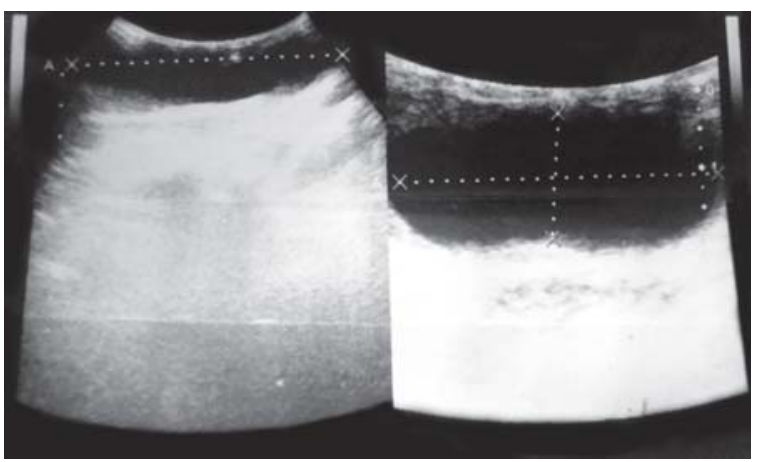

Fig. 2: Cystic lesion in posterior triangle of neck measuring $8.6 \times 4.0 \times 1.5 \mathrm{~cm}$ (volume $28 \mathrm{ml}$ ) with well-defined margins and few septations

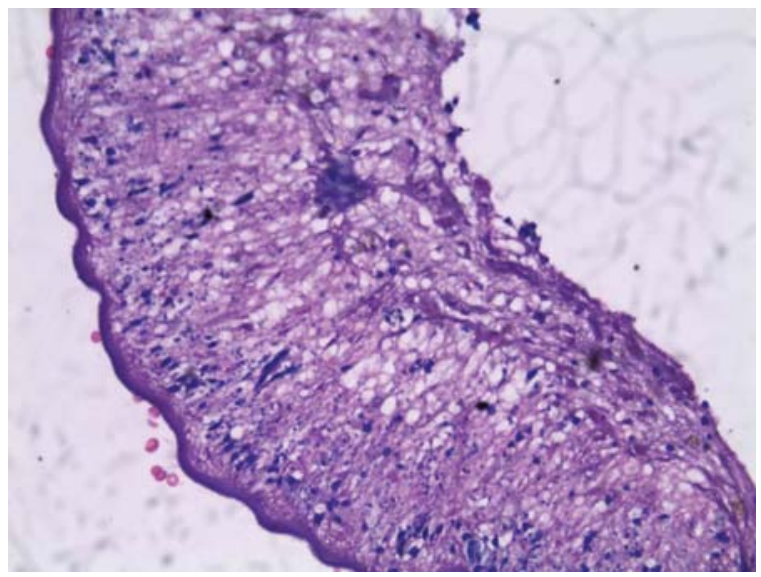

Fig. 3: Histopathology showing cyst wall with thick cuticle layer and underlying fibrovascular stroma (H\&E 400x)

T. solium, the pork tapeworm, can cause two distinct types of infection. The type of infection that develops depends on whether humans are infected with adult tapeworms in the intestine or with larval forms in the tissues, so called cysticercosis. Tapeworm eggs hatch and embryos penetrate the intestinal wall and disseminate to different tissues via systemic circulation. Neurocysticercosis is the most common type of manifestation which is diagnosed when multiple cystic ring enhancing parenchymal lesions are detected on contrast-enhanced computed tomography (CT). In head and neck manifestation, cysticercosis can present as soft tissue mass at the submental area, cheek and oral tongue. Soft tissue cysticercosis presents in the form of a painless swelling of long-term duration. Specific investigations used in diagnosis of cysticercosis include CT scan, MRI and ultrasonography. ${ }^{5}$ Generally, radiological studies are mandatory to rule out central nervous system involvement. ${ }^{6} \mathrm{CT}$ scan and MRI are useful in demonstrating the location, size and number of cysts along with occasional pathognomonic features of cysticercosis lesion like scolex or sucking parts of the larva. ${ }^{7}$ Because of its wide availability, ultrasonography can be the preferred initial modality for evaluation of superficial masses. On high resolution ultrasonography cysticercosis are seen as well- defined anechoic or hypoechoic lesions with or without calcification with a hyperechoic area within it suggestive of scolex. ${ }^{8}$ Ultrasonography is sensitive for detection of ocular cysticercosis and may be used for guided fine needle aspiration cytology (FNAC). Cysticercosis presenting as a neck mass is diagnosed by microscopic examination in which $T$. solium larvae are found. Definite diagnosis is by the identification of detached hooklets, scolex and fragments of spiral wall of cysticercosis. In some cases section smears show no larval parts but contain inflammatory reaction consisting of large number of eosinophils and palisading histiocytes which is suggestive of a parasitic cyst. The management of cysticercosis involves surgery and supportive medical treatment. Surgery is usually feasible in cases of isolated intramuscular or subcutaneous cysticercosis lesions, which may otherwise require prolonged course of antiparasitic therapy. In cases of cervical lymphadenopathy, wide local excision of the involved surrounding soft tissue should be included in surgery.

\section{REFERENCES}

1. Jain RK, Gupta OP, Aryya NC. Cysticercosis of the tongue. J Laryngol Otol 1989;103:1227-28.

2. Gupta SC, Gupta SC. Cysticercosis of the tongue. Ear Nose Throat J 1995;74:174-78.

3. Tripathy SK, Sen RK, Sudes P, Dhatt S. Solitary cysticercosis of deltoid muscle in a child: Diagnostic dilemma and case report. J Orthopaedics 2009;6(2):e11.

4. Garcia HH, Del Brutto OH. Taenia solium cysticercosis. Infect Dis Clin North Am 2000;14:97-119.

5. Hawk MW, Shahlaie K, Kim DK, Theis JH. Neurocysticercosis: A review. Surg Neurol 2005;63:123-32.

6. Sawhney IM, Sigh G, Lekhra OP, Mathuriya SN, Parihar PS, Prabhakar S. Uncommon presentations of neurocysticercosis. J Neurol Sci 1998;154:94-100.

7. Sirikulchayanonta V, Jaorsidha S. An intramuscular cysticercosis: A case report with correlation of Magnetic Resonance imaging and histopathology. J Med Assoc Thai 2007;90(6): 1248-52.

8. Mittal A, Gupta S, Gupta S, Mehta V. Subcutaneous and intramuscular cysticercosis: High-resolution sonography. Indian J Dermatol Venereol Leprol 2009;75:515-16.

\section{ABOUT THE AUTHORS}

\section{Sunil Kumar (Corresponding Author)}

Assistant Professor, Department of ENT and Head and Neck Surgery King George’s Medical University, Lucknow, Uttar Pradesh, India e-mail: drsunil_kumar123@rediffmail.com

\section{SP Agarwal}

Professor and Head, Department of ENT, King George's Medical University, Lucknow, Uttar Pradesh, India

\section{Malti Kumari}

Lecturer, Department of Pathology, King George’s Medical University Lucknow, Uttar Pradesh, India 